\title{
Analysis of Compliance and Barriers for Hand Hygiene Practices among Health Care Workers during COVID-19 Pandemic Management in Tertiary Care Hospital of India-An Important Step for Second Wave Preparedness
}

\author{
Keshvi Chauhan, Yogita Mistry, Summaiya Mullan \\ Government Medical College, Surat, India \\ Email: mullasummaiya@gmail.com
}

How to cite this paper: Chauhan, K., Mistry, Y. and Mullan, S. (2020) Analysis of Compliance and Barriers for Hand Hygiene Practices among Health Care Workers during COVID-19 Pandemic Management in Tertiary Care Hospital of India-An Important Step for Second Wave Preparedness. Open Journal of Medical Microbiology, 10, 182-189.

https://doi.org/10.4236/ojmm.2020.104016

Received: October 7, 2020

Accepted: November 27, 2020

Published: November 30, 2020

Copyright $\odot 2020$ by author(s) and Scientific Research Publishing Inc. This work is licensed under the Creative Commons Attribution International License (CC BY 4.0).

http://creativecommons.org/licenses/by/4.0/

\section{Abstract}

Introduction: Hands of health care workers play an important part in infection transmission in a healthcare setting infections. Even though it is a simple and effective procedure, it is many times ignored by the health care workers (HCWs) due to lack of knowledge and interest, time constraints, skin irritation, understaffing, etc. There are no specific drugs and vaccines available for COVID-19 pandemic management, so as control measures, hand hygiene is an essential measure. It will be helpful even for the second wave preparedness. So this study was planned for analysis of barriers and practices of hand hygiene among health care workers which determine the adherence of hand hygiene in medical practice. Methods: This was a cross-sectional study designed during COVID-19 pandemic management to analyze the barriers and knowledge, attitude and practices in health care workers for hand hygiene. Semi-structured questionnaires based study based on previous experience of seasonal flu outbreak was make to know barriers as well as to analyze the knowledge, attitude and practices of Health care workers. 215 participants were enrolled who were doctors, resident doctors, nurses, lab technicians, ward boys and food distributors. Results: The "Barriers" related questions' analysis showed that hand hygiene facilities, with the supply of soap, hand rub, basins with adequate as well as clean water, are available at various places in the Hospital. In knowledge related questions, major gaps are found in 5 moments of hand hygiene and regarding aspects of alcohol-based hand-rub. Majority HCWs show a good attitude towards questions that hand hygiene is 
an important thing to do along with other works and they feel frustrated when others or they themselves omit hand hygiene. But the attitude toward hand hygiene when hands are not visibly soiled and when working with gloved hand showed a wrong attitude. There is a major gap in practices of hand hygiene. The majority feel hand hygiene is difficult due to work pressure, sometimes they forgot, they feel it difficult to adhere due to more steps during an emergency. Discussion: Hand hygiene is an essential part of COVID-19 pandemic management. Less compliance was observed due to a lack of knowledge and awareness. Such ignorance leads to an increase in Hospital-acquired infection. Conclusion: Good preparedness by Government and Institute for supplying adequate hand hygiene facilities in the work area for health care workers. But there were few gaps found in knowledge and attitude-related questionnaires and major gaps in practices were found which should be corrected by repeated training, awareness programs, demonstrations for hand hygiene and hands-on training. Conducting Periodic Webinar can be the most useful, convenient and safe method to provide training during the COVID-19 pandemic.

\section{Keywords}

Hand Hygiene, COVID-19, Barriers, Health Care Workers, Knowledge, Attitude, Practices

\section{Introduction}

Novel coronavirus SARS-CoV-2 has recently been detected (December 2019) amid an outbreak of a cluster of cases of pneumonia of unknown etiology in Wuhan city, Hubei province, China. Within the next $8-12$ weeks, it spread globally to the extent that the World Health Organization (WHO) declared it as a global pandemic on March 11, 2020 [1]. SARS-CoV-2 like the other coronaviruses has a lipid envelope and in simple words, washing with soap can break that fat in the envelope apart and thereby making it impossible or difficult for the virus to spread. Hands of health care workers (HCWs) play an important part in infection transmission in a healthcare setting. Poor adherence to hand hygiene practices is responsible for $40 \%$ of nosocomial infections. Even though it is a simple and effective procedure, it is many times ignored by the health care workers due to lack of knowledge, interest, time constraints, skin irritation, understaffing, etc. [2] [3]. As there is no specific treatment or vaccine available to date, hand hygiene is an important step to prevent the spread of infection in present scenario and also as an important step for second-wave preparedness of COVID-19. So to bring about positive change in hand hygiene behaviour, it is first important to understand the hurdles leading to poor compliance. Hence, this study was planned to understand and compare the knowledge, attitude and practices related to hand hygiene among HCWs and to identify the barriers which affect the adherence of hand hygiene in health care workers. 


\section{Material \& Methods}

This was a cross-sectional study designed during COVID-19 pandemic management at a tertiary care hospital of India to analyze the barriers and knowledge, attitude and practices in health care workers for hand hygiene. Semi-structured questionnaire-based study based on our previous experience of seasonal flu outbreak [4]. Health care workers both medical and paramedical staff working in COVID-19 pandemic management were targeted for the study. Approximately 300 health care workers were working in COVID-19 management at a tertiary care Hospital in India. Considering the non-response rate of $25 \%$ due to a busy schedule, non-consent, working with personal protective equipment (PPE) approximately 215 participants enrolled. Convenient sampling was done for those willing to participate and who comprehend Gujarati/English [5]. Treating doctors, resident doctors, nurses, lab technicians, ward boys, food distributors were included. Participants were enrolled during their break time or before entering or leaving duty hours. Every participant was interacted with individually. They were informed briefly about the study by participant information sheet as well as informed that only 10 minutes are needed at once for filling up questionnaires. Informed consent was taken from every participant. Complete questionnaires about hand hygiene were given to every participant. The identity of them was decoded by giving them unique ID numbers. Human Research Ethical Committee of the institute approved the study design. The survey was done during June 2020. All collected data was entered in an excel sheet. Scoring was done as 0 for a wrong answer and 1 for the right answer for barrier, knowledge and practices related questions. For attitude-related question analysis Likert type of grading was used for the answer of strongly agree, agree, neutral, disagree and strongly disagree. SPSS software version 12 was used to analyze the statistical data. $\mathrm{P}$-value of less than 0.05 was used to see the statistical significance level [6].

\section{Result}

During June 2020, the study was conducted after ethical approval. During that time, health care workers/frontline workers who were working in COVID-19 and who were willing in COVID 19 study were enrolled. Overall 215 health care workers were enrolled out of which $28 \%$ of faculty and resident doctors, $12 \%$ Intern doctors, $14 \%$ were Lab Technicians, $29 \%$ were nurses, $17 \%$ were servants. Out of 215, 27 healthcare workers had past experience of work during a seasonal flu outbreak (H1N1, etc.) of which 12 were doctors, 4 were nurses, 2 servants and 9 lab technicians were there.

Regarding barriers related questions of hand hygiene, 59 (98\%) teaching faculty and resident doctors, 25 (96\%) Intern doctors, 30 (97\%) Laboratory technicians, 57 (92\%) Nurses, 24 (67\%) servants had received training related to hand hygiene. Hand hygiene facilities were available at workplace as per 47 (78\%) teaching faculty and resident doctors13 (50\%) Intern doctors, 30 (97\%) Laboratory technicians, $26(42 \%)$ Nurses, $24(67 \%)$ servants. Sufficient amount of 
handwashing solutions and hand rubs were available as per 46 (75\%) teaching faculty and resident doctors, 8 (31\%) Intern doctors, 29 (94\%) laboratory technicians, 26 (42\%) nurses, 24 (67\%) servants. Sufficient numbers of washbasins with running water available as per $45(75 \%)$ teaching faculty and resident doctors, 7 (27\%) Intern doctors, 31 (100\%) Laboratory technicians, 24 (39\%) Nurses, 25 (69\%) servants. Proper cleanliness is maintained at handwashing area as per $44(73 \%)$ teaching faculty and resident doctors, 10 (39\%) Intern doctors, 30 (97\%) Laboratory technicians, 24 (39\%) Nurses, 24 (67\%) servants. Training programs were conducted by the infection control team related to hand hygiene as per $49(82 \%)$ teaching faculty and resident doctors, 11 (42\%) Intern doctors, 31 (100\%) Laboratory technicians, 26 (42\%) Nurses, 25 (69\%) servants.

Figure 1 is showing the total number of health care workers with correct responses for knowledge related questionnaires. Regarding steps of handwashing question (K1) was answered correctly by $51(85 \%)$ teaching faculty and resident doctors, 13 (50\%) Intern doctors, 25 (81\%) laboratory technicians, 38 (61\%) nurses, $16(44 \%)$ servants with p-value 0.0001 . Hand hygiene is recommended before the medical examination, before taking blood samples, before touching patients, after touching patients, after touching linen or bedding of patients, after wound dressing (K2) is answered correctly by $45(75 \%)$ teaching faculty and resident doctors, 7 (27\%) Intern doctors, 27 (87\%) laboratory technicians, 38 (61\%) nurses, 22 (61\%) servants. Minimum time required for alcohol-based hand rub (K3) is answered correctly by 28 (47\%) teaching faculty and resident doctors, 9 (35\%) Intern doctors, 5 (16\%) laboratory technicians, 19 (31\%) nurses, and 10 (28\%) servants. The likelihood of colonization of hands (K4) is increased with wearing jewelry, damaged hands, use of artificial nails, etc was known and answered correctly by $54(90 \%)$ teaching faculty and resident doctors, 23 (89\%) Intern doctors, 30 (97\%) Laboratory technicians, 51 (82\%) Nurses, 21 (58\%) servants with p-value 0.0002. Regarding alcohol-based hand rub (K5) uses, effectiveness related question was answered correctly by 53 (88\%) teaching faculty and resident doctors, 21 (81\%) Intern doctors, 29 (94\%) laboratory technicians, 41 (66\%) nurses, and 18 (50\%) servants.

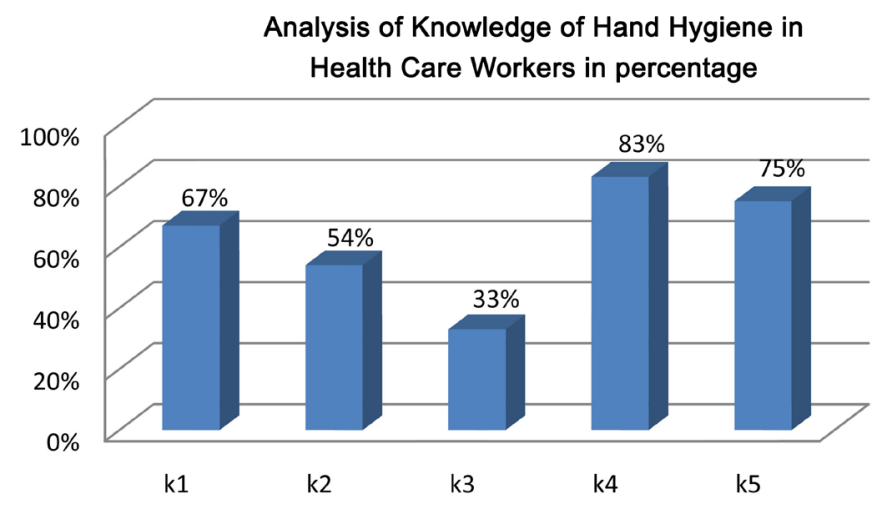

Figure 1. Knowledge related questions analysis showing No. of HCWs with correct response. 
Attitude related questionnaires analysis of all health care workers is shown in Figure 2. 68 Health care workers disagree with the question that they have sometimes more important things (A1) to do than hand hygiene with p-value 0.002. $125 \mathrm{HCWs}$ were in agreement to the questions that they feel frustrated when they omit the hand hygiene (A4) or when other omits hand hygiene (A3) with p-value 0.005 . For other attitude related questions like wearing gloves reduces the need for hand hygiene (A2), hand hygiene can be omitted if hands are not visibly soiled (A5) have a correct partial response.

Regarding practices related questions HCW analysis is as per Figure 3. Adhering to hand hygiene practices with proper steps is easy without much efforts or problems (P1) as per $42(70 \%)$ teaching faculty and resident doctors, 20 (77\%) Intern doctors, 30 (97\%) Laboratory technicians, 50 (81\%) Nurses, 31 (86\%) servants with p-value 0.0006. Emergencies during COVID-19 pandemic management make hand hygiene practices sometimes difficult (P2) as per $48(80 \%)$ teaching faculty and resident doctors, 22 (85\%) Intern doctors, 26 (84\%) laboratory technicians, 40 (65\%) nurses, 22 (61\%) servants. Hand hygiene were missed out sometimes as they forgot (P3) to follow is answered by 29 (48\%) teaching faculty and resident doctors, 14 (54\%) Intern doctors, 1 (3\%) laboratory technicians, 24 (39\%) nurses, 12 (33\%) servants with p-value 0.0002. Hand hygiene is an essential part during COVID-19 pandemic management (P4) as per 56 (93\%) teaching faculty and resident doctors, 25 (96\%) intern doctors, 30 (97\%) laboratory technicians, 59 (95\%) nurses, 31 (86\%) servants. Frequency of handwashing makes it difficult to adhere (P5) as per $36(60 \%)$ teaching faculty and resident doctors, 15 (58\%) intern doctors, 17 (55\%) laboratory technicians, 32 (52\%) nurses, 5 (14\%) servants with p-value 0.0002 . Work pressure makes hand hygiene difficult to do (P6) as per $30(50 \%)$ teaching faculty and resident doctors, $12(46 \%)$ intern doctors, 2 (7\%) laboratory technicians, 31 (50\%) nurses, 20 (57\%) servants with p-value 0.0002 .

\section{Analysis of Attitude for hand hygiene among health care workers in percentage}

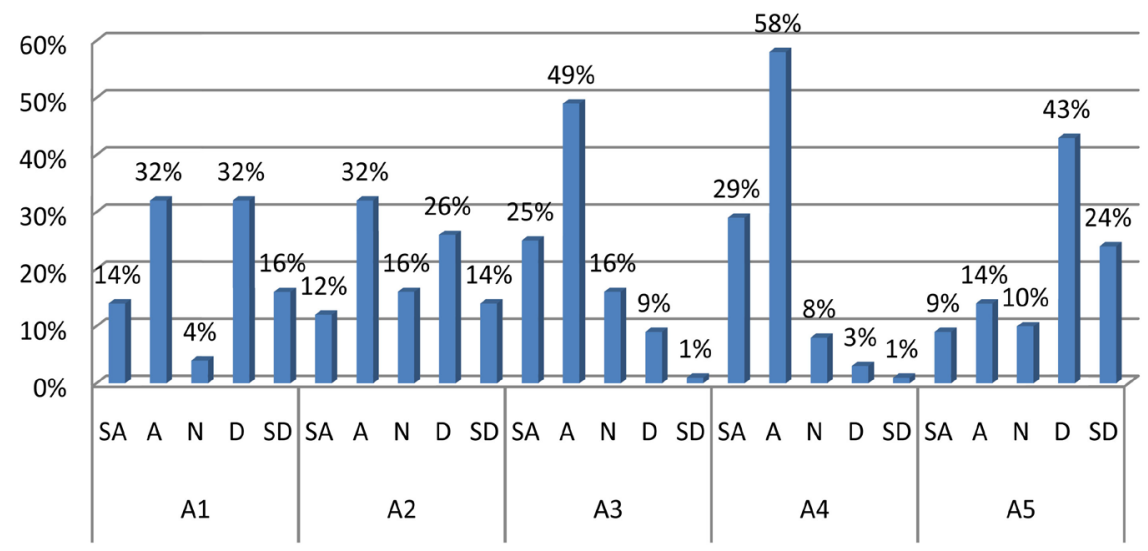

Figure 2. Attitude related questions analysis of 215 HCWs. 
Analysis of Practice of hand hygiene among

health care workers in percentage

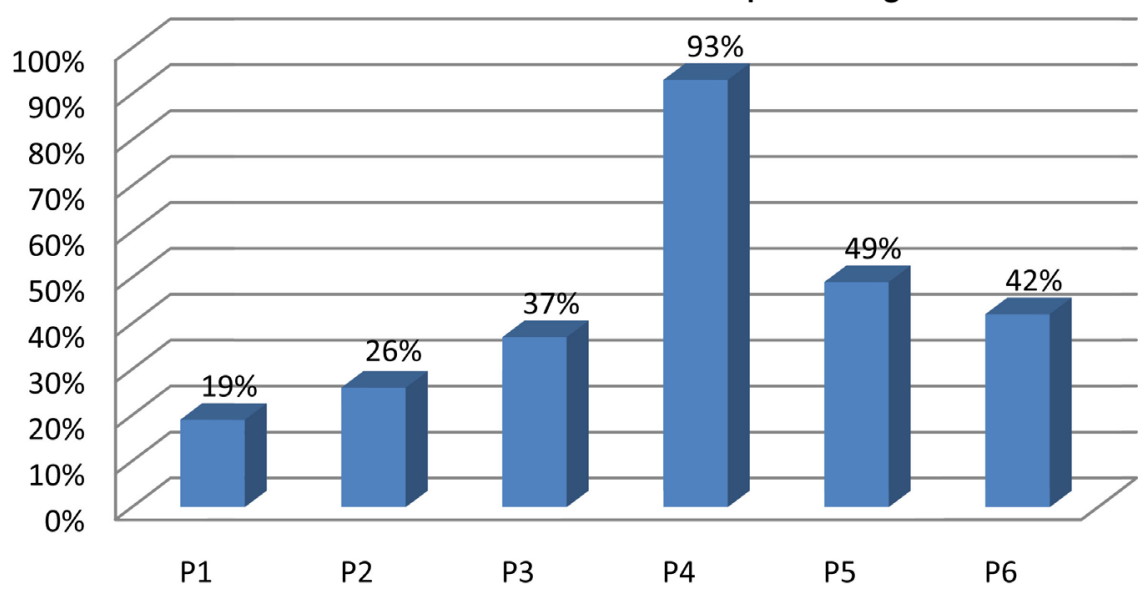

Figure 3. Practice related Questions analysis of $215 \mathrm{HCWs}$ with the correct response.

\section{Discussion}

Hospital-acquired infection prevention and control during COVID-19 pandemic management relies on self preparedness by Health care workers which is analyzed in this study by knowledge, attitude and practice related questionnaires, and institutional preparedness which is analyzed by barrier questions to HCWs [7].

Barriers related questions of hand hygiene suggest that overall institutional preparedness is good for providing hand hygiene facilities, sufficient solutions of hand wash and hand rubs available, washbasins with running tap water available, proper training by infection control team conducted for hand hygiene, proper cleanliness available at hand hygiene facilities [8]. For knowledge related questions, overall partial response was found with major gaps found in servants and Interns doctors. Overall knowledge regarding steps of hand hygiene is good and knowledge for colonization of hands with germs increases with the use of jewellery, damaged hands, use of artificial nails, etc is well known in health care workers [9]. But knowledge regarding the minimum time required for alcohol-based hand rub use and other aspects for hand rub show poor knowledge. 5 moments of hand hygiene that is before touching the patient, after touching the patient, after touching patient samples, after wound dressing, after touching patients linen, etc show poor response [10]. Until complete adherence with hand hygiene cannot be followed by health care workers, one cannot get rid of Infection and infection spreads to society and hospital [11]. These findings are comparable to other studies [7] [8].

Regular classes \& training programs are conducted for nursing personnel as a part of Infection control practice as shown in pooja et al. [9]. This suggests a need for repeated training as the staff may be newly appointed, especially for servants and interns doctors.

Attitude related questions suggest that overall attitude towards hand hygiene 
during COVID-19 pandemic management is good in the majority of Health care workers. Majority HCWs show a good attitude towards questions which state hand hygiene is an important thing to do along with other works and it should not be ignored due to work pressure, they feel frustrated when others omit hand hygiene or feel guilty when they omit hand hygiene. But the attitude toward hand hygiene when hands are not visibly soiled and when working with gloved hand show the wrong attitude, due to the myth that hands will remain clean if they are not visibly soiled or when they are gloved hands. So these suggest that they are not following hand hygiene practices properly. Such gaps in attitude questions are reflected.

But there is a major gap in practices of hand hygiene. The majority feels hand hygiene is difficult due to work pressure, sometimes they forgot, they feel difficulty in adhering to many steps in emergency situations. They know that hand hygiene is essential during COVID-19 pandemic management, but they are not practicing due to lack of habit and knowledge. In the present time, it was observed that Hand hygiene adherence will be helpful to reduce hospital-acquired infection and to reduce the morbidity and mortality of patients and for this COVID-19 pandemic where no specific drugs or vaccine is available, this can be the only option to prevent the spread of infection and also for preparedness for the second wave of COVID-19 infection [10] [11].

\section{Conclusions}

Health care workers working during COVID-19 pandemic management in tertiary care Government hospital of South Gujarat is having a good preparedness of response by the Government and Institute, but there is a gap found in knowledge and attitude and major gaps in practices of healthcare workers, which should be corrected by repeated training, awareness programs and demonstrations for hand hygiene.

Conducting Periodic Webinar, Zoom meetings, Video demonstrations and reminders, etc. can be the most useful, convenient and safe method to provide training during the COVID-19 situation.

\section{Conflicts of Interest}

The authors declare no conflicts of interest regarding the publication of this paper.

\section{References}

[1] Sahu, K.K., Mishra, A.K. and Lal, A. (2020) Comprehensive Update on Current Outbreak of Novel Coronavirus Infection (2019-nCoV). Annals of Translational Medicine, 8, 11. https://doi.org/10.21037/atm.2020.02.92

[2] Pittet, D. and Boyce, J.M. (2001) Hand Hygiene and Patient Care: Pursuing the Semmelweis Legacy. The Lancet Infectious Diseases, 1, 9-20. https://doi.org/10.1016/S1473-3099(09)70295-6

[3] Wendt, C. (2001) Hand Hygiene-Comparison of International Recommendations. 
Journal of Hospital Infection, 48, S23-S28. https://doi.org/10.1016/S0195-6701(01)90008-8

[4] Lippi, G., Adeli, K., Ferrari, M., Hovarth, A.R., Koch, D., Sethi, S., et al. (2020) Biosafety Measures for Preventing Infection from COVID-19 in Clinical Laboratories: IFCC Taskforce Recommendations. Clinical Chemistry and Laboratory Medicine, 58, 1053-1062. https://doi.org/10.1515/cclm-2020-0633

[5] Houghton, C., Meskell, P., Delaney, H., Smalle, M., Glenton, C., Booth, A., et al. (2020) Barriers and Facilitators to Healthcare Workers' Adherence with Infection Prevention and Control (IPC) Guidelines for Respiratory Infectious Diseases: A Rapid Qualitative Evidence Synthesis. Cochrane Database of Systematic Reviews, Article No. CD013582. https://doi.org/10.1002/14651858.CD013582

[6] Malhotra, N., Gupta, N., Ish, S. and Ish, P. (2020) COVID-19 in Intensive Care. Some Necessary Steps for Health Care Workers. Monaldi Archives for Chest Disease, 90, 161-162. https://doi.org/10.4081/monaldi.2020.1284

[7] Godoy, L.R.G., Jones, A.E., Anderson, T.N., et al. (2020) Facial Protection for Healthcare Workers during Pandemics: A Scoping Review. BMJ Global Health, 5, e002553. https://doi.org/10.1136/bmjgh-2020-002553

[8] Powell-Jackson, T., King, J.J.C., Makungu, C., Spieker, N., Woodd, S., Risha, P., et al. (2020) Infection Prevention and Control Compliance in Tanzanian Outpatient Facilities: A Cross-Sectional Study with Implications for the Control of COVID-19. The Lancet Glob Health, 8, E780-E789. https://doi.org/10.1016/S2214-109X(20)30222-9

[9] Sarmah, P., Hemavathi, Rajashekar, S., et al. (2016) Knowledge, Attitude and Practices of Hand Hygiene among MBBS Students and Nursing Personnel. Journal of Evolution of Medical and Dental Sciences, 5, 2083-2086. https://doi.org/10.14260/jemds/2016/489

[10] Modi, P.D., Nair, G., Uppe, A., Modi, J., Tuppekar, B. and Gharpure, A.S. (2020) COVID-19 Awareness among Healthcare Students and Professionals in Mumbai Metropolitan Region: A Questionnaire-Based Survey. Cureus, 12, e7514. https://doi.org/10.7759/cureus.7514

[11] Vivian, H.Y., Sondekoppam, R.V., Ozelsel, T.J.P. and Tsui, B.C.H. (2020) COVID-19 Pandemic: International Variation of Personal Protective Equipment and Infection Prevention and Control Guidelines. Anesthesia \& Analgesia, 131, e113-e114. 